\title{
The Loading Curve of Spherical Indentions Is Not a Parabola and Flat Punch Is Linear
}

\author{
Gerd Kaupp \\ University of Oldenburg, Oldenburg, Germany \\ Email: gerd.kaupp@uni-oldenburg.de
}

How to cite this paper: Kaupp, G. (2019) The Loading Curve of Spherical Indentions Is Not a Parabola and Flat Punch Is Linear. Advances in Materials Physics and Chemistry, 9, 141-157.

https://doi.org/10.4236/ampc.2019.98012

Received: July 29, 2019

Accepted: August 27, 2019

Published: August 30, 2019

Copyright (อ 2019 by author(s) and Scientific Research Publishing Inc. This work is licensed under the Creative Commons Attribution International License (CC BY 4.0).

http://creativecommons.org/licenses/by/4.0/ (c) (i) Open Access

\begin{abstract}
The purpose of this paper is the physical deduction of the loading curves for spherical and flat punch indentations, in particular as the parabola assumption for not self-similar spherical impressions appears impossible. These deductions avoid the still common first energy law violations of ISO 14577 by consideration of the work done by elastic and plastic pressure work. The hitherto generally accepted "parabolas" exponents on the depth $h$ ("2 for cone, $3 / 2$ for spheres, and 1 for flat punches") are still the unchanged basis of ISO 14577 standards that also enforce the up to $3+8$ free iteration parameters for ISO hardness and ISO elastic indentation modulus. Almost all of these common practices are now challenged by physical mathematical proof of exponent $3 / 2$ for cones by removing the misconceptions with indentation against a projected surface (contact) area with violation of the first energy law, because the elastic and inelastic pressure work cannot be obtained from nothing. Physically correct is the impression of a volume that is coupled with pressure formation that creates elastic deformation and numerous types of plastic deformations. It follows the exponent $3 / 2$ only for the cones/pyramids/wedges loading parabola. It appears impossible that the geometrically not self-similar sphere loading curve is an $h^{3 / 2}$ parabola. Hertz did only deduce the touching of the sphere and Sneddon did not get a parabola for the sphere. The radius over depth ratio is not constant with the sphere. The apparently good correlation of such $h_{\text {sphere }}^{2 / 3}$ parabola plots at large $R / h$ ratios and low $h$-values does not withstand against the deduced physical equation for the spherical indentation loading curve. Such plots are unphysical for the sphere and so tried regression results indicate data-treatments. The closed physical deduction result consists of the exponential factor $h^{3 / 2}$ and a dimensionless correction factor that is depth dependent. The non-parabola against force plot using published data is concavely bent even for large radius/depth-ratios at the shallow indents. The capabilities of conical/pyramidal/wedged indentations are thus lost. These facts are outlined for experimental nano- and micro-indentations.
\end{abstract}


Spherical indentations reveal that linear data regression is suspicious and worthless if it does not correspond with physical reality. This stresses the necessity of the straightforward deductions of the correct relations on the basis of iteration-less and fitting-less undeniable calculation rules on an undeniable basic physical understanding. The straightforward physical deduction of the flat punch indentation is therefore also presented, together with formulas for the physical indentation hardness, indentation work, and applied work for these geometrically self-similar indentations. It is exemplified with a macroindentation.

\section{Keywords}

Closed Formula for Spherical Indentation, Challenge of ISO14577, Mathematical Proofs, Volume Instead of Area, Correct Flat Indentations, Physical Indentation Hardness, Hardness Dependence on Indenter Shape, Data Treatment Detection

\section{Introduction}

The most severe misconception in the powerful (nano) indentation field is the two-dimensional treatment of the three-dimensional impression into solids. Such behavior became highly applauded world-wide since 1992. It seemed to be an iron rule for indentations that the force-depth curves for conical, spherical, flat punch indenters are parabolas with the exponent 2, 3/2, and 1 (finally straight), respectively, and it is still the basis of ISO 14577 [1] [2] [3]. This theory is worldwide accepted and applauded in academia [1], textbooks [2] and industry [3]. They still use a "parabola with exponent $3 / 2$ for spheres", well knowing that the spherical indentation is not "geometrically self-similar" (ratio of impression radius or diagonal over depth is not constant). Furthermore it is used for defining the size of their always used correction factor " $\mathcal{E}$ " [4], for their iteration of the projected contact area according to the work by [1], and by ISO 14577 for "refining" their ISO-hardness $\left(H\left[\mathrm{~N} / \mathrm{m}^{2}\right.\right.$ or GPa]) and ISO-elastic modulus $E$ calculations. These include false exponent and energy-law violation and undue "Young's modulus" claim by ([1], etc.) and ISO 14577, as first published in [5] [6]. Spherical indentions cannot physically be described by a $F_{\mathrm{N}}$ $h^{3 / 2}$ parabola ( $F_{\mathrm{N}}=$ normal force, formerly often called "P"), even though experimental plots of $h^{3 / 2}$ versus $F_{\mathrm{N}}$ appear to be linear for high $R / h$ values (sphere radius over depth) and low depth ranges.

However, a physical background was missing. H. Hertz deduced an equation with exponent $3 / 2$ for the mathematical touching of a sphere and a flat surface or a second sphere, and for horizontally sliding of solid bodies without pressure [7]. Hertz himself literally stressed the validity of his deduction in [7] only for the mathematical touching (not indenting) at one single point in [8], and Sneddon's solution for spheres is not a parabola [9]. Nevertheless, the unproved parabola 
has also been used for the determination of the tip rounding of pyramidal indenters. Uncountable AFM (atomic force microscopy) publications reported on the "spherical Hertzian exponent 3/2" without citing Hertz work and without giving their equation for the impression contact under force. No force-depth relation with exponent $3 / 2$ can be found in [8] and Hertz's hardness definition does not help either. This can not at all be taken as a description of indentations under load. In addition, the authors of [1] and ISO 14577 took over the force-less contact for spherical indentations under force for indentations. Also the textbook [2] explained the inconstant a/ $R$ ratio ( $R=$ indenter radius, $a=$ radius of the impression, Figure 1) for not self-similarly penetrating spheres in its Figure 1.4 for angles between 0 and at least $\pm 33^{\circ}$ from the normal line. The author did not consider that such angle would not be proportional to the depth of spherical indentations. Users were nevertheless taught that they can go deeply onto the surface with such a parabola. Interestingly, while the exponent 2 for conical indents of Sneddon [9] (his pre-exponential constant differed from the one of Love [10]) and exponent 1 for flat indents were welcomed in [1] and by ISO 14577, but Sneddon's non-parabola solution for spheres with after all three members and three different exponents on $h(2,1$, and 0$)$ after rewriting of his formula was disregarded (inappropriate [7] was preferred). One just took what is liked and disregarded what is disliked for the sphere in [9]. However, we do not agree with the solution of Sneddon for spheres as the false premises of [9] were the same as those that ended with the false exponent 2 for cones.

Also its solution for flat punches is only valid with respect to the correct exponent.

The universal correct exponent for cones is in fact $3 / 2$ as undeniably proved in [11] on the basis of sacrosanct calculation rules. This is also not in accord with the still common "iron rule" of ISO etc. Unfortunately, the "iron rule" was not

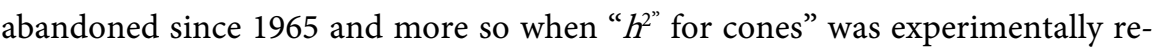
placed by $h^{3 / 2}$ in 2004 [12], even though the elegant and simple physical deduction (finally published in 2016 [11]) was ripe for deduction. But the false exponent "2" for cones/pyramids/wedges is still defended by biased anonymous reviewers who fight against the $F_{\mathrm{N}}=k h^{3 / 2}$ plot for conical/pyramidal/wedged indentations and unduly call it "Kaupp-fitting" (it must therefore now be called Kaupp-plot). Furthermore, they use and cite easily traced juggler tricks from published papers revealing unbelievable lack of mathematical knowledge of very basic calculation rules. These include unequal dimension on both sides of equations, or not realizing that the proportionality factor of loading parabolas has a dimension that depends on the exponent of $h$ as does its value, or by defining indentation hardness as force over surface area, and then claiming a "theoretical confirmation of $h^{2 \prime}$ for the cone and pyramid, as deduced from such definition. Unfortunately, this includes ISO 14577 officials, authors, editors, and biased anonymous peer reviewers. Nevertheless, ISO 14577 and numerous recent publications still claim the so-called Hertzian exponent on a "parabola with exponent $3 / 2$ " on $h_{\text {sphere. }}$ Others simply claim to use an unspecified "Hertzian theory" 


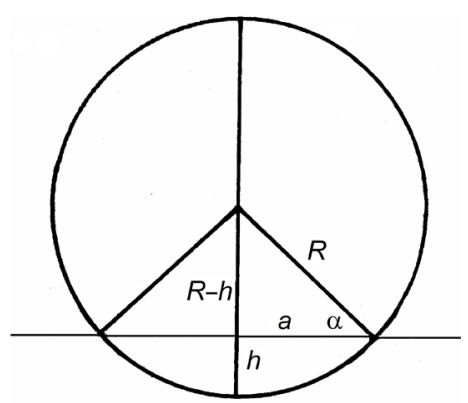

Figure 1. Schematic representation of a sphere partly immersed beneath an initial plane surface with an angle $\alpha$ that is differently defined as the one mentioned in Section 1 of [2].

but without citing the original (e.g. [7] and [8]) and not telling what they mean with that, when spheres are penetrating into flat surfaces.

Before having the here disclosed solution the present author expressed however on his worldwide lectures the opinion that the spherical loading curves might at best only approximate the exponent $3 / 2$ on $h$ for experimental spherical indentations, for large $R / h$ ratios and shallow indents [1] [2], ISO 14577. This speculation deserves the straightforward physical deduction, because $h^{3 / 2}$ cannot at the same time be valid for geometrically self-similar cones/ pyramids/wedges and the not self-similar sphere [11]. But even the present author obtained good correlations with $F_{\mathrm{N}}=k h^{3 / 2}$ plots in the analyzed spherical indentations from the literature. He added the word "apparently" to this exponent of a parabola [13] and that these slopes cannot be compared with the ones from pyramids [13]. But the reported $k_{1}$-value from the nickel-superalloy (published $R=269 \mathrm{~nm}$ ) [14] and the $k$-value from PDMS ((polydimethylsiloxane) $(R=192 \mu \mathrm{m})([15]$, in [13] are no longer penetration resistances, because the now deduced Equation (7) excludes a parabola for spherical indentation. $F_{\mathrm{N}}=k h^{3 / 2}$ plots for spherical indentations are physically in error, irrespective of the linear correlations with their large radius/dept ratios and narrow depth ranges. This will be shown in this paper. We deduce in this work the physical load-depth behavior for spheres on the same basis as the deduction in [11] by Kaupp with respect to the impressed volume but not with respect to a projected contact area, and check both of these correct and incorrect approaches. By doing so, we will also complete the story with the physical proof of the (correct) exponent 1 for flat punch indentations. A consistent theoretical understanding is achieved. Both spherical indentations were reanalyzed in Section 3.2.2. and 3.2.3.

Fortunately, the unprecedented new results did not affect our elegant and correct calculation of the PDMS adhesion energy with $0.5 F_{\mathrm{N}} h$ in [5], which corrected the complicated JKR (Johnson, Kendall, Roberts) [16] iterative process in [15] by a factor of 2.66 [5].

\section{Materials and Methods}

The indentation onto a hard metallic nickel superalloy with a blunt Berkovich 
indenter with claimed end radius of $269 \mathrm{~nm}$ was selected, because its calculated cone depth $\left(h_{\text {cone }}=R(1-\sin \beta)\right.$ [13]) of $15.75 \mathrm{~nm}$ was very close at $15.9 \mathrm{~nm}$. The final depth of only $50.3 \mathrm{~nm}$ allowed for the safe collection of 13 data pairs after a minor surface effect. Conversely, the data of a soft PDMS sample (polydimethylsiloxane) in combination with a huge spherical indenter radius and providing both 54 "experimental" and 54 fitted data pairs at considerable depths were calculated and printed with Excel. The data collections were from the digitized published loading curves with the Plot Digitizer 2.5.1 program; http://www.softpedia.com/. The calculations of Equation (7) used a pocket calculator with 10 figures to avoid rounding errors and the results reasonably rounded in the Tables and text.

\section{Results and Discussion}

\subsection{The Force-Depth Parabola of Conical/Pyramidal/Wedged Indentations}

We repeat here the physical deduction of the exponent for the loading parabola of self-similar conical, pyramidal and wedged indenters to remind the elegant straightforward technique. Indentations create two connected processes: the volume formation and the total pressure [11]. This has hitherto been disregarded or ignored, even though the retained part of the pressure (not transformed for plasticizing) has been amply used from the beginning for the elastic modulus iteration from the unloading curve by [1], ISO 14577. It remained unconsidered that even elastic pressure creates work that is generated by the force. We have therefore to start with a normal force $\left(F_{\mathrm{N}}\right)$ parabola $F_{\mathrm{N}}=k h^{x}$ with two components, one for volume $(V)$ and the other for pressure $(p): F_{\mathrm{N}}=F_{\mathrm{N}-V}^{m} \cdot F_{\mathrm{N}-p}^{n} \quad(1)$. When doing so we have to consider that the total pressure $\left(p_{\text {total }}\right)$ (remaining pressure plus loss of pressure in case of all of the different modes of plastic conversions) must be used. The exponents $\mathrm{m}$ and $\mathrm{n}$ must sum up to 1 for obtaining $F_{\mathrm{N}}$. As $p_{\text {total }}$ is undoubtedly proportional to the indented volume of the indenter under the originally flat surface we have for the cone with its mathematical volume at the depth $h$ the equation $p_{\text {total }}=K V=K \pi(\tan \alpha)^{2} h^{3} / 3$. The conversion of $h r^{2}$ into $(\tan \alpha)^{2} h^{3}$ is self-evident. Similarly, we have $h^{3}$ also for the volumes of pyramids and wedges. As $p_{\text {total }}$ is proportional to $h^{3}$ also $F_{\mathrm{N}-p} \propto h^{3}$, and $h$ relates to $F_{\mathrm{N}-p}^{1 / 3}$, which is lost for the indentation depth, but it tells us that $F_{\mathrm{N}-V}^{m}=F_{\mathrm{N}-V}^{2 / 3}$. So we have physically on the basis of arithmetic calculation rules $F_{\mathrm{N}-V}^{2 / 3}=$ const $h$ or $F_{\mathrm{N}-V}=k h^{3 / 2}$ (Equation (1)) [11] where $k$ denotes the chemo-physical properties of the material in question. This describes in detail the deduction of the universal exponent $3 / 2$ for conical/pyramidal/wedged indentations with hard indenters (diamond), independent of the materials, as could be finally published in 2016 (2).

$$
\begin{gathered}
F_{\mathrm{N}}=F_{\mathrm{N}-V}^{m} \cdot F_{\mathrm{N}-p}^{n} \\
F_{\mathrm{N}-V}=k h^{3 / 2}
\end{gathered}
$$




\subsection{The Force-Depth Curve of Spherical Indentations}

\subsubsection{Theoretical Considerations, Deduction of Equation (7)}

The spherical indentation is generally assumed (ISO14577, [1] [2] [3] [4]) to occur as parabola with exponent $3 / 2$. The non-indentation but only touching deduction of Hertz [7] [8] was taken as theoretical background, but there is concern, whether cones/pyramids/wedges could have the same exponent for loading parabolas. Experimentally it appeared that such behavior would be also valid for spherical indentations, but only for large $R / h$ values and the low penetrations that are achieved. Also the present author Kaupp obtained good correlations with $F_{\mathrm{N}}=k h^{3 / 2}$ plots from the published "spherical" indentations that he analyzed, but the word "apparently" was added to this spherical exponent [13] and an initial approximation was claimed. As already mentioned, Sneddon [9] did not get a parabola at all, but this deduction was disregarded in [1] [2] [3] [4]. The solution of Sneddon is as follows when his equation "6.13" is substituted in "6.15" on page 54 of [9]. By now using the common letters for the corresponding subjects one obtains $F_{\mathrm{N}}=[E /(1-v)]\left[\left(a^{2}+R^{2}\right) 2 h / a-a R\right]$ with strongly varying $h / a-$ ratio $=\operatorname{cotg} \beta$ (for $\beta$ cf. Figure 1 ) as a three-membered "solution". From there with $a=h / \cot \beta$ one obtains $F_{\mathrm{N}}(1-v) / E=2 h^{2} / \cot \beta-h R / \cot \beta+2 R^{2} \cot \beta$. This is not a parabola but $\cot \beta$ is variable ( $E=$ "Young's" modulus, $v=$ Poisson's ratio). Experimental results are not in agreement with the solution of Sneddon, as it depends on his undue premises (area instead of volume) that also led to the disproved exponent 2 for cone/ pyramid/wedge. The physical solution is now deduced.

When starting with using volume instead of area as shown in Section 3.1, we reformulate the mathematical volume of the immersed calotte (3A) by multiplication with $1=h / h$ and obtain the form of (3B), which separates $h^{3}$ and a dimensionless though $h$-dependent correction factor. Further forms are (3C) with two different exponents on $h$ and finally for the angle $\alpha$ dependence (3D). According to Figure $1(R-h) / R=\sin \alpha$, so that $h / R=(1-\sin \alpha)$ or $R / h=1 /(1-\sin \alpha)$. This can be substituted in (3B) to obtain (3D), so that one can also check the angle ranges of the depressions. All 4 forms A-D of (3) are equal.

$$
\begin{gathered}
V=\pi h^{2}(R-h / 3) \\
V=\pi h^{3}(R / h-1 / 3) \\
V=\pi h^{2} R-\pi h^{3} / 3 \\
V=\pi h^{3}[1 /(1-\sin \alpha)-1 / 3]
\end{gathered}
$$

We first show how much the dimensionless volume factor of (3D) changes for various angles $\alpha$ between $30^{\circ}$ (very deep impression) and $89.5^{\circ}$ (very flat impression) in Table 1 . They indicate the strong non-linear variation of the volume factor with the indention depth.

Table 1 clearly shows the enormous variation of the dimensionless factor for Equations (3B), which excludes a physical parabola exponent for spherical 
Table 1. Formal analysis of the volume factor $\pi[1 /(1-\sin \alpha)-1 / 3]=\pi(R / h-1 / 3)$ at varying angle $\alpha$.

\begin{tabular}{cccc}
\hline$\alpha$ & $\sin \alpha$ & $1-\sin \alpha$ & $\pi[1 /(1-\sin \alpha)-1 / 3]$ \\
\hline 30 & 0.5000 & 0.5000 & 0.5236 \\
40 & 0.642787609 & 0.35721239 & 7.7475 \\
50 & 0.766044443 & 0.23395556 & 13.4282 \\
60 & 0.866025403 & 0.13397460 & 23.4492 \\
70 & 0.93969262 & 0.06030738 & 52.0930 \\
80 & 0.984807753 & 0.01519225 & 207.7429 \\
89.5 & 0.999961923 & 0.00003808 & $82,499.8071$ \\
\hline
\end{tabular}

indentation load-depth curves. It is only valid for every single point of such curve with its own $R / h$ ratio in accord with the physical deduction in Section 3.1, because the point by point changing pre-exponential dimensionless factor multiplies with the penetration resistance $k_{\text {sphere }}\left[\mathrm{N} / \mathrm{m}^{3 / 2}\right]$ (cf. (2)). The claimed parabolas seem to be excluded under these conditions. There is the proviso that so named "experimental data" could have been somehow iterated and fitted. We do not invoke the sphere quality here. It is clearly seen at (3), that the calotte volume is not only described by $h^{3}$ as we had it with the cone (pyramid, wedge) where we deduced the exponent $3 / 2$ on $h$ in Section 3.1. There is now a dimensionless factor that changes with the $R / h$ ratio (3B).

The physical deduction of the spherical loading curve using (3B) starts with the evidently coupled processes of volume formation and pressure formation. As in Section 3.1 it means $F_{\mathrm{N}}=F_{\mathrm{N}-V}^{m} \cdot F_{\mathrm{N}-p}^{n}$ (1). Both factors relate to the immersed volume and $n+m$ must give 1 (1). The total pressure ( $p_{\text {total }}$ is the sum of $p$ that remains $+p$ for all plastic deformations) has to be considered, as it leads to reversible and to plastic deformations. It is without any doubt that $p_{\text {total }}$ is proportional to the impressed volume $V$. Thus $p_{\text {total }} \propto h^{3} \pi(R / h-1 / 3)$ and thus also $F_{\mathrm{N}-p}$ are proportional to $\pi h^{3}(R / h-1 / 3)$ (4). So we obtain the exponent $\mathrm{n}=1 / 3$ when $h$ is expressed in (5) and $\mathrm{m}=2 / 3$ because both exponents must add to 1 . As the pressure part of the force is lost for the impression, it follows that $F_{\mathrm{N}-V}^{2 / 3}$ is proportional to $h \pi^{2 / 3}(R / h-1 / 3)^{2 / 3}$ (6), and thus for the impression $F_{\mathrm{N}-V}=k h^{3 / 2} \pi(R / h-1 / 3) \quad$ (7) where $k$ takes care of the relevant chemo-physical properties of the material in question. $F_{\mathrm{N}-\mathrm{v}}$ controls the depth and can now be abbreviated as $F_{\mathrm{N}}$ in relation to the depth) (7) of the not self-similar spherical indenter. The calculation of $\alpha$ indicates the angle range of the experimental loading curves. Equation (7) shows that the penetration resistance $k \pi(R / h-1 / 3) \quad\left[\mathrm{N} / \mathrm{m}^{3 / 2}\right]$ of spherical indentations is not constant and it cannot be easily compared with the $k$-values of pyramidal indentations. The latter are normalized for their cone angles [13]. The physical deduction uses the undeniable fact that the indenter volume is immersed into the material and that the force is in part used for pressure stress that produces elastic and plastic work. This pressure stress is not part 
of the depth formation that determines the immersed indenter volume. Earlier deductions [9] did not care for the pressure part and they thus violated the first energy law [1] [2] [3] and ISO 14577. Such unphysical "deductions" were mathematically very complicated [9] [15] [16] and yielded false results. The physical deduction in this work does not need more than basic calculation rules in (1) and (3) through (7) for the spherical indentation with its inconstant penetration resistance $\left(k \pi(R / h-1 / 3)\left[\mathrm{N} / \mathrm{m}^{3 / 2}\right]\right)$ with its materials factor $k$ and the geometrical factor $\pi(R / h-1 / 3)$ that also depends on the depth. Equation (1) is also the start point for the spherical indentation.

$$
\begin{gathered}
F_{\mathrm{N}}=F_{\mathrm{N}-V}^{m} \cdot F_{\mathrm{N}-p}^{n} \\
p_{\text {total }} \text { and thus also } F_{\mathrm{N}-p} \propto \pi h^{3}(R / h-1 / 3) \\
F_{\mathrm{N}-p}^{1 / 3} \propto h \pi^{1 / 3}(R / h-1 / 3)^{1 / 3}
\end{gathered}
$$

With $n=1 / 3$ and $m=2 / 3$ it follows that $F_{\mathrm{N}}^{2 / 3}$ is proportional to the depth $h$, and with the $h$-dependent dimensionless geometrical factor $\pi(R / h-1 / 3)$ one obtains:

$$
F_{\mathrm{N}-V}^{2 / 3} \propto h \pi^{2 / 3}(R / h-1 / 3)^{2 / 3}
$$

After rewriting and inclusion of the materials factor $k$ one obtains:

$$
F_{\mathrm{N}}=k h^{3 / 2} \pi(R / h-1 / 3)
$$

The closed Equation (7) is the physical description of the loading curve for spherical indentations. However, there are further experimental reports telling that the exponent $3 / 2$ describes spherical indentation parabolas sometimes without reliable knowledge of the conospherical radius. And there are rather useless "spherical indentations" when so called "effective radii" are continuously changed along the loading curve with power-law fitting [17]. We therefore analyze only "experimental" measurements without such and other fittings or iterations. These appear to be the loading curves of a Ni-based superalloy with nominal largely rounded Berkovich up to the cone-point of $15.9 \mathrm{~nm}$ and a PDMS nominal experimental spherical indentation. Both exhibit large $R / h$ ratios that decrease rapidly with the depth $h$. By doing so we compared the now disproved parabola approach using the Kaupp plot of Equation (2) [5] [11] [13] and the physical loading curve Equation (7) for comparison purposes. The results are collected in Table 2 and Table 3, respectively, in Sections 3.2.2 and 3.2.3.

We have to stress here, that the adhesion energy by jump below the surface of PDMS or similarly in force experiments with AFM, as $0.5 F_{\mathrm{N}} h$ for the negative $F_{\mathrm{N}}$-region stays valid independent of the loading curves' shape [5] and that it continues to correct the erroneous JKR iterations, as e.g. in [15] (Section 3.2.3).

\subsubsection{The Presumed "Spherical" Nickel-Superalloy Indentation}

Spherical indentations can be obtained with blunted conical/pyramidal indentations below the cone point depth, when the depth in the following "cone region" is only short. A typical example is the nickel-superalloy loading curve from [14]. 
Table 2. Analysis of a Ni-based superalloy indentation of a blunt Berkovich up to the cone point depth as it would be with $R=269 \mathrm{~nm}$ ) according to (7).

\begin{tabular}{ccccccc}
\hline$h(\mathrm{~nm})$ & $h^{3 / 2}$ & $R / h$ & $\pi(R / h-1 / 3)$ & $h^{3 / 2} \pi(R / h-1 / 3)$ & $F_{\mathrm{N}}(\mu \mathrm{N})$ & $\alpha\left(^{\circ}\right)$ \\
\hline 0.17728 & 0.07464 & 1517.37 & 4765.91126 & 355.7276 & 5.5039 & 87.9198 \\
0.94430 & 0.91763 & 284.867 & 893.888878 & 820.2593 & 15.1171 & 85.1978 \\
2.74338 & 4.43902 & 98.0542 & 306.999157 & 1362.7753 & 32.2638 & 81.8102 \\
3.83473 & 7.50935 & 70.1484 & 219.330501 & 1647.0295 & 43.9297 & 80.3140 \\
4.95573 & 11.0322 & 54.2806 & 169.480337 & 1869.7410 & 57.6617 & 78.9850 \\
5.87044 & 14.2235 & 45.8228 & 142.909374 & 2032.6715 & 71.4031 & 78.0081 \\
6.81462 & 17.7894 & 39.4740 & 122.296389 & 2175.5794 & 85.1433 & 77.0758 \\
7.84709 & 21.9818 & 34.2802 & 106.647301 & 2344.2996 & 100.51 & 76.1268 \\
8.88041 & 26.4636 & 30.2914 & 94.1160423 & 2489.0205 & 116.75 & 75.2368 \\
9.82479 & 30.7953 & 27.3797 & 84.9686669 & 2616.6356 & 135.004 & 74.4671 \\
10.9166 & 36.0687 & 24.6414 & 76.3661338 & 2754.4427 & 153.183 & 73.6211 \\
11.7138 & 40.0910 & 22.9644 & 71.0975929 & 2850.3736 & 165.001 & 73.0293 \\
12.5993 & 44.7218 & 21.3504 & 66.0270623 & 2952.8491 & 178.254 & 72.3947 \\
\hline
\end{tabular}

Table 3. Analysis of a spherical indentation onto PDMS with a Borosilicate glass ball $(R=$ $192 \mu \mathrm{m})$ of [15], according to Equation (7).

\begin{tabular}{ccccccc}
\hline$h(\mu \mathrm{m})$ & $h^{3 / 2}$ & $R / h$ & $\pi(R / h-1 / 3)$ & $h^{3 / 2} \pi(R / h-1 / 3)$ & $F_{\mathrm{N}}(\mathrm{mN})$ & $\alpha\left(^{\circ}\right)$ \\
\hline 0.0 & & & & & -0.02759 & 90 \\
0.4 & 0.2529822 & 480 & 1506.91729 & 381.2232 & -0.00919 & 86.3009 \\
0.8 & 0.7155418 & 240 & 752.93505 & 538.7565 & 0.019923 & 84.7678 \\
1.2 & 1.3145341 & 160 & 501.60764 & 659.3803 & 0.055012 & 83.5908 \\
1.6 & 2.0238577 & 120 & 375.9448 & 760.8588 & 0.094993 & 82.5980 \\
2.0 & 2.8284271 & 96 & 300.54640 & 850.0736 & 0.141720 & 81.7229 \\
2.4 & 3.7180640 & 80 & 250.28022 & 930.5579 & 0.248921 & 80.9313 \\
2.8 & 4.6852962 & 68.57 & 214.37182 & 1004.3955 & 0.239081 & 80.2030 \\
3.2 & 5.7243340 & 60 & 187.44837 & 1073.0171 & 0.298851 & 79.5247 \\
3.6 & 6.8305198 & 53.33 & 166.49395 & 1137.2402 & 0.350958 & 78.8873 \\
\hline
\end{tabular}

The determined $F_{\mathrm{N}}$ and $h^{3 / 2}$ data below the cone point are listed in Table 2. The published end radius of the Berkovich indenter was $269 \mathrm{~nm}$. After removal of the initial effects the $R / h$ ratios are from 284.867 to 21.350 . The value of $h_{\text {cone }}=R(1-\sin \beta)$ [13] is $15.75 \mathrm{~nm}$, where $\beta$ is the effective cone angle of the Berkovich. The observed $h_{\text {cone }}$ is at $15.9 \mathrm{~nm}$, which corresponds closely to the calculated value in apparent accord with a spherical indentation part. The Kaupp-plot for the postulated parabola (not shown) gives a straight line with a slope of 3.873 $\mu \mathrm{N} / \mathrm{nm}^{3 / 2}\left(\mathrm{r}^{2}=0.9997\right)$. This can however not be a confirmation of the $h^{3 / 2}$ parabola for spheres, because the physical correction factor of $\pi(R / h-1 / 3)$ in (7) has not 
been applied. The physically correct $F_{\mathrm{N}}$ versus $h^{3 / 2} \pi(R / h-1 / 3)$ plot (7) (Table 2) with the same data points gives the concavely bent loading curve of Figure 2. The comparison of these different outcomes is difficult, but we try with a provisional trendline for the Figure 2 curve, the slope of which calculates to 0.0688 $\mu \mathrm{N} / \mathrm{nm}^{3 / 2}$. This gives at least a rough hint for the enormous error (despite the high correlation) when the unphysical parabola assumption ([1] and ISO14577) for spherical indentations is applied. However, the change of $R / h$ (Table 2) or $\sin \alpha$ (Table 1) is undeniable and the hard to explain apparent "parabolas" bear the risk that their slopes be interpreted as being real for further applications. The first goals of indentations are hardness and elastic modulus and when these are unphysical, their errors are perpetuated in the there from defined further characterizations of the materials. For example 12 different applications of the indentation modulus from viscoelasticity to fracture toughness are listed in [18].

The apparent slopes of unreal spherical indentation parabola plots cannot be interpreted as physical indentation hardness and used for face transition energies and activation energies as in the case of conical/pyramidal/wedged indentations (Section 3.1). Also the $k$-value of this nickel-superalloy sample that was published in [13] is invaluable. It is no longer the penetration resistance for spherical indentations. It would suggest far too high constant hardness instead of the changing hardness when the depth increases. The reason is the particular formula (3) for the penetration calotte volume, even in the very narrow ranges. One has to use Equation (7) for a correct result. The authors of [14] unduly called their cone point at $15.9 \mu \mathrm{m}$ a "pop-in" "marking the transition from elastic to plastic deformation". When $R$ would be $269 \mathrm{~nm}$ it would however be the change from spherical to pyramidal indentation. There is however the proviso: this kink would most likely indicate a harder surface layer with about $15 \mathrm{~nm}$ height on the bulk, provided that the pyramidal end-radius was actually sharp. This tip-radius might have been determined as the second free parameter $\left(C_{1}\right.$ with the dimension of a length) of the second iterated term $\mathrm{C}_{1} \mathrm{~h}_{\mathrm{c}}$ from the eight parameter iteration of the contact area from [1] and ISO 14577. This technique is often performed and leads to undue very large "radii", so that this is the most likely explanation as a parabola from a sharp pyramid. There is almost no opposition against this tip rounding iteration technique in the literature in addition to [13] [19] [20]. Tip radii must not be iterated but measured with tapping mode AFM [13]. This indentation should be repeated with a certified sharp Berkovich indenter to finally clarify these points. Table 2 and Figure 2 contain the results showing the shape of the plot that would describe the spherical indentation when $R$ is indeed $269 \mathrm{~nm}$ (7). It clearly confirms the non-parabola for such spherical indentation.

\subsubsection{The Spherical PDMS Indentation}

The result with the Ni-superalloy requires also the analysis of a much deeper indentation with a huge sphere radius and much deeper penetration with very compliant materials. A published nominal "experimental" PDMS spherical 


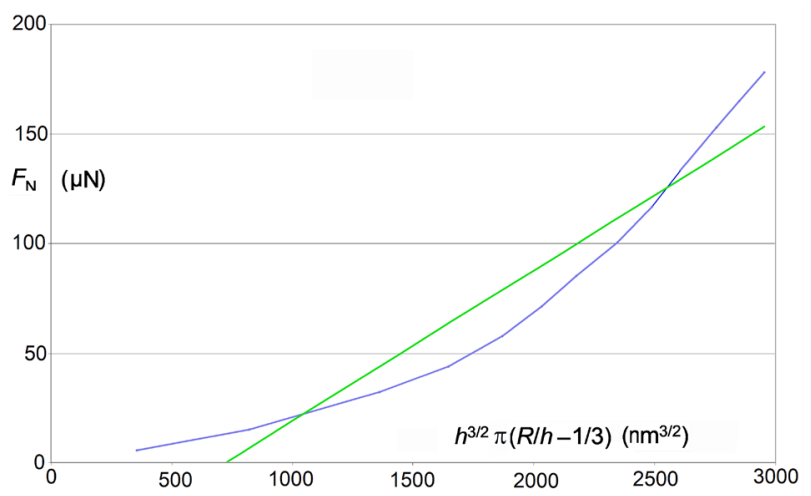

Figure 2. $F_{\mathrm{N}}$ versus $h^{3 / 2} \pi(R / h-1 / 3)$ plot (7) of a Ni-based superalloy with a rounded Berkovich (claimed $R=269 \mathrm{~nm}$ ) after the removed initial effect (first point at $30 \mu \mathrm{N}$ load) up to the cone point at $180 \mu \mathrm{N}$; the green line is the provisional trendline (only for comparison) with a slope of 0.2169 $\mu \mathrm{N} / \mathrm{nm}^{3 / 2}$.

indentation (the sphere radius was $R=192 \mu \mathrm{m}$ ) and the JKR iterated and fitted unloading curve [15] were analyzed for comparison. Both provide $F_{\mathrm{N}}-h^{3 / 2}$ parabolas against (7). The jump below the surface requires starting from the so formed negative force minimum, but the adsorption energy does not disturb the analyses [5] [13]. The $R / h$ ratio varies from 480 to 53.33 (Table 3). Also the present author had analyzed these loading curves with respect to the claims of ISO 14577 and [1] [2] [3] [4] by applying the Kaupp-plot. Kaupp published apparently well correlating $k$-values [5] [13] that can however no longer be considered as being valid, because they violate the physical deduction of the closed formula (7) (Section 3.2.1). The slope of the (unphysical) $F_{\mathrm{N}}-h^{3 / 2}$ data (not shown here) was $0.04912 \mathrm{mN} / \mu \mathrm{m}^{3 / 2}$ ( $r=0.9999$ with 54 data points). Also for the JKR-fitted unloading curve the corresponding correlation with a slope of 0.0560 $\mathrm{mN} / \mu \mathrm{m}^{3 / 2}$ calculated with $r=0.9999$. This appears to be a good fit, but it does not prove anything, because it does not correspond with the physical Equation (7). We try to explain these features against physical evidence and analyze the experimental loading curve with Equation (7) in Table 3 and Figure 3. There remains the proviso that the loading curve named "experimental" was perhaps also JKR iterated and fitted to correspond with the prescribed parabola of [1] and ISO 14577. Again there are increasing values of $h^{3 / 2}$ multiplied with decreasing correction factor values. The plot according to (7) is again concavely bent. Comparison can again only be tried with the provisionally calculated trendline in Figure 3. Its slope is $0.0005 \mathrm{mN} / \mu \mathrm{m}^{3 / 2}$. The so judged error of the unphysical parabola is almost twice as much as in the nickel-superalloy in Section 3.2.2. Such JKR-iterations and fittings are detrimental treatments of experimental data, as already challenged in [5]. Here the JFK iterations and fittings calculated what the authors wanted to see: an "iron-rule" parabola with exponent $3 / 2$ as prescribed by ISO 14577 and ([1], etc.), which is clearly disproved by (7). Our provisional green trendline does not mean that the original experimental data would lie close to it or on it. Iterated and fitted data cannot be reconstructed. 


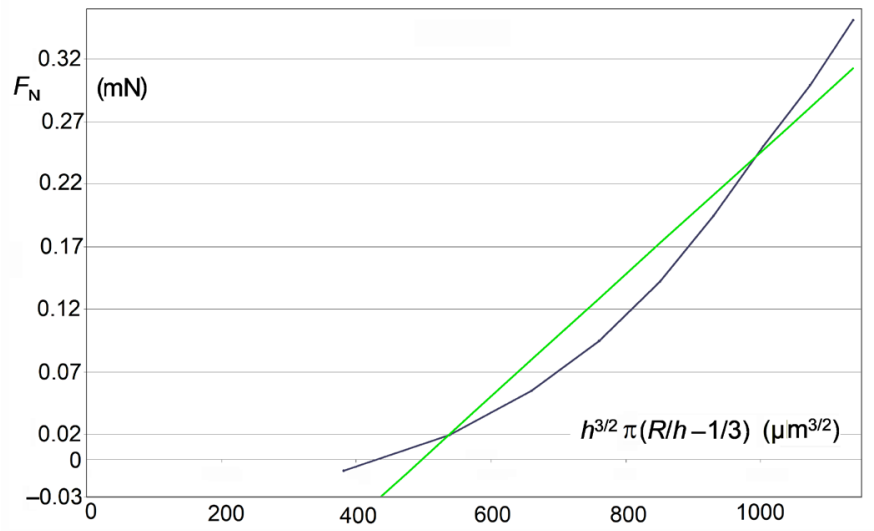

Figure 3. $F_{\mathrm{N}}$ versus $h^{3 / 2} \pi(R / h-1 / 3)$ plot of the "experimental data" of the PDMS polymer with a Borosilicate glass ball $(R=192 \mu \mathrm{m})$ [15] according to (7); it starts at the minimum after jump below the surface; the green line is the provisional trendline (only for comparison) with a slope of $0.0005 \mathrm{mN} / \mu \mathrm{m}^{3 / 2}$.

Our physical analysis is important, because any non-physical direct plot $F_{\mathrm{N} \text {-sphere }}$ versus $h^{3 / 2}$ would give vastly different false indentation hardness values as already exemplified with the Ni-based superalloy in Section 3.2.2. Clearly, spherical indentations are unable to obtain hardness values. They would change from depth to depth for every $R / h$-value (Table 3 ). Also the hitherto used older formula of [1] (" $S=2 E_{\mathrm{r}} A^{1 / 2} / \pi^{1 / 2}$ ”) is incorrect for spherical indentations.

It must be noted here that our AFM (atomic force microscopy) loading curve of polystyrene with a silicon cantilever (typical apex radii $10-15 \mathrm{~nm}$ ) in [13] [19] is the result of a pyramidal indentation, but not of a "spherical indentation". After an initial effect well below $100 \mathrm{~nm}$ depth the pyramidal indentation proceeded down to $550 \mathrm{~nm}$ depth with $16 \mu \mathrm{N}$ load.

\subsection{The Force-Depth Relation of Flat Cylinder or Beam Indentations}

It is widely accepted that the flat indenter (either column or beam) proceeds linearly ([1] [2] [3] and ISO 14577). According to Sneddon the cylinder penetrates with $F_{\mathrm{N}}=2 r h E /(1-v)^{2}$ [9] (here $r$ as column radius, $h$ depth, $E$ Young's modulus. and $v$ Poisson's ratio). The exponent on $h$ is thus 1 , but we need a clear-cut deduction without Young's modulus avoiding the premises of Sneddon. This has however never been studied before. As there is also the volume formation coupled to pressure formation one starts the deduction as in Section 3.1, which tells that one does not indent towards a projected indenter face, but against the volume formation by the indenter. Any almost negligible compression of the very hard indenter is part of the pressure action. The volume of the cylinder $V=\pi r^{2} h$ or of the beam $V=a^{2} h$, where a means the edge, has only $h$ as the variable. In that situation both the pressure and the immersed volume are both directly proportional to the depth. The pressure part is again lost for the depth and we get directly $F_{\mathrm{N} \text {-flat }}=k_{\text {flat }} h^{1}$ (8). The applied force and thus 
work is the area of the $F_{\mathrm{N}}-h$ triangle (9). It is 1:1 divided between pressure work $W_{\text {flat-pressure }}$ and the indentation work $W_{\text {flat indent }}$. The latter is thus the area of the $F_{\mathrm{N}}-h$ triangle (9). $W_{\text {flat-indent }}$ is thus $1 / 4 F_{\mathrm{N}-\max } h_{\max }(10)$, again in accordance with the first energy law [5] [6]. The penetration resistance $k_{\text {flat }}$ is here the physical indentation hardness with the dimension $[\mathrm{N} / \mathrm{m}]$, which is also the full flat loading stiffness $(S)$. And $k_{\text {flat }} / 2$ is the energy corrected flat impression hardness = stiffness. The definition of [1] ( $S=2 E_{\mathrm{r}} A^{1 / 2} / \pi^{1 / 2}$ ”) is also here in error, because it relies on the unsuitable premises of Sneddon by using area instead of volume [9].

$$
\begin{gathered}
F_{\mathrm{N}-\text { flat }}=k_{\text {flat }} h \\
W_{\text {flat-applied }}=1 / 2 F_{\mathrm{N}-\max } h_{\text {max }} \\
W_{\text {flat-indent }}=1 / 4 F_{\mathrm{N}-\max } h_{\text {max }}
\end{gathered}
$$

Load versus depth curves of flat punches onto visco-elastic materials seem to be rare, as creep and moduli were the prevailing points of interest. However, load-depth curves with cylinder of $0.5 \mathrm{~cm}$ radius onto $\mathrm{F} 82 \mathrm{H}$ steel at different temperatures $\left(92^{\circ} \mathrm{C}\right.$ to $\left.-196^{\circ} \mathrm{C}\right)$ used loads up to $600 \mathrm{~N}$ and were linear up to 100 $\mathrm{N}$ at about $30 \mu \mathrm{m}$ depths. They continued at first with moderate bending up to $250 \mathrm{~N}$ and further so up to $600 \mathrm{~N}$ towards $1 \mathrm{~mm}$ depth. The first part up to about $50 \mathrm{~N}$ or $0.5 \mathrm{GPa}$ with a poorly resolved kink was reported as "fully reversible". The kink was followed linearly up to $150-200 \mathrm{~N}$ or 1.5 - $2 \mathrm{GPa}$, depending on the temperature. This experimentally confirms the indentation law $\left(h_{\text {flat }}^{1}\right)$ for the low resolved initial parts. The bending above these loadings was interpreted with three different plastic stages [21]. As such bending is not in accord with (8) it has to be concluded that the experimental control was lost at such high loads, most probably by macroscopic undetected cracks and perhaps chemical transformations of the different components. The following bending starts with protruding of material around the imprint or chemical reactions [21]. Regular pile-up would not destroy the physical law (8) [5] [22]. Interestingly, these results are comparable with the yield stress of tensile tests, the values of which are about 3 times smaller than the stress at the start of the bending for 16 tested metallic materials [21]. The elastic moduli have been obtained from the slopes of the initial linear part of the unloading curves from $120-110 \mu \mathrm{m}$ without iterations. But energy law corrections that should be the factor 0.5 according to (9) and (10) [5] [6] have not been considered. The obvious comparability of flat macro-indentation with tensile tests appears interesting for further applications.

\section{Continuation}

The presented deductions of physical equations for conical/pyramidal/wedged, spherical, and flat indentations must urgently replace the false historical equations of ISO 14577 with their energy law violations for obtaining reliable mechanical parameters and to enable the new applications (detection of phase-transition onsets, their transition energies and activation energies) that were previously not 
possible. Further applications are to be developed particularly at very low temperatures for airplanes and spacecraft vehicles. The spherical indentations are the most complicated and least rewarding ones. The spherical work of indentation by integration of Equation (7) does not provide a rational ratio with the total work of indentation, as comparable with the 4/5 ratio in the case cones/pyramids [5]. This detracts from the possibility for the important energetic evaluations and the not self-similar spherical indentations are thus inferior to the indentations with the self-similar indenters. The same is true for paraboloids of revolution for which [1] falsely claimed loading parabolas with exponent $3 / 2$. They are centrosymmetric but not self-similar. Also ellipsoids and two-sheet-hyperboloids of revolution are centrosymmetric but not self-similar indenters that would lack their advanced use.

The possibilities of flat punch indentation require further development and promise interesting further applications beyond the creep determinations. ISO must be further urged to thoroughly revise and modernize ISO 14577 on the physical basis without iterations and data-fittings. This will remove the falsely obtained mechanical materials parameters; which is of urgent importance for avoiding the risks with technical products in daily life. The certification of industrial producers must be based on physical reality rather than on historical errors to enable the use of the correct novelties and the development of improved materials in a correct way, so that catastrophic mechanical failures can be avoided or at least minimized. Responsibilities can no longer be shifted to ISO 14577 and from there to historical researchers.

\section{Conclusions}

The most severe misconception in the powerful (nano) indentation field has been reported in this paper. It is the two-dimensional treatment of the three-dimensional impression into solids, with its now obsolete "iron-rule" ("cone exponent 2, sphere exponent $3 / 2$, and flat exponent 1 "). The main problem was the impossible equality of exponent $3 / 2$ for conical/pyramidal [11] and for spherical indentation loading curves [1] [2] [3]. This had to be solved despite the common ISO 14577 standards. Only the exponent 1 for flat punch indentation was not debated, but it also required the deduction of a correct Equation (8). By prescription of [1] and ISO 14577 conical parabola should have the exponent 2 on $h$ instead of $3 / 2$. Therefore, faithful researchers tried to question the universality of $h^{3 / 2}$ for cones/pyramids/wedges and iterated huge tip rounding with 8 free parameters for indenters, while proposing exponent $3 / 2$ parabolas for spheres. By doing so these researchers had to iterate and fit their data from spherical indentation for example with JKR techniques, in order to obtain treated data providing four-nines-correlations that exhibited what they expected to observe. Both detrimental techniques (iteration of excessive tip radii and iterative data fitting treatments) are uncovered in this work. It could be done because the undeniably deduced physically formulas cannot be overcome. Also CSM Instruments Ap- 
plication Bulletin 35 advocates for FEA (finite element models) and JKR iterations for spherical indentations on the basis of $h^{3 / 2}$ parabolas. The enormous errors of such iterative and fitting data treatments are roughly judged. Another iteration and fitting technique is the continuous change of "effective radii" along the loading curve with power-law fitting [17]. It is particularly bothersome that the materials are falsely calculated (including violation of the energy law) and that phase-transitions under load cannot be traced with false exponent and shape of the untreated loading curves. The correct value of the exponents is of utmost importance for increased precision by regression, the calculation of materials' properties, the recognition of initial surface effects, gradients, and phase transitions with their transition energies and activation energies [5] [13] [22] [23] [24] [25]. The therein and in this paper challenged behaviors create high risks and miss the important possibilities for avoiding catastrophic failures by grain formations that initiate catastrophic failures within the polymorph interfaces [22]. All of that is inaccessible with the unphysical exponents and iterations with fittings. The differentiation between loading parabolas, non-parabolas, and straight lines is also important. The physical deductions of the loading curves use the volume of the indenters, rather than their surface area or "contact area", to obtain closed formulas without any iterations and data fittings in a very elegant and simple way as compared to previous work. A comprehensive understanding has been achieved now for conical/pyramidal/wedged, spherical, and flat-punch indentations. This has been shown for experimental nano- and micro-indentations. Further applications are expected for AFM studies of visco-elastic-plastic biological and medical preparations. The universal exponent $3 / 2$ loading parabola is only valid for cones/pyramids/wedges [11]. Spherical indentations provide non-parabola loading curves according to the now deduced Equation (7), and flat-punch indentation with exponent 1 follow the new Equation (8). The reason for the non-parabola at spherical indentations is the geometrically not self-similar impression of sphere calottes.

Clearly, undeniable closed physical formulas based on sacrosanct calculation rules stands before regression analysis of a physically incorrect parabola. This shows that any good linear correlations without physical background are a handle to detect data-treatments. Sorry to say: it might perhaps be easier for mainstream researchers, who do not dare to challenge ISO 14577, to please it and its proponents.

The new unprecedented physical deductions rely on the indented volume (not on projected area) by using undeniable calculation rules [11]. All of the errors from the previous falsely believed (including violation of the energy rule) indentation exponents are to be abandoned. The still exacting of the world by the common ISO 14577 standards with the energy law violations derived from the unsuitable mathematical premises of [1] [2] [3] [4] [9] [10] that did not consider that the "depth formation work" (projected area instead of volume) is coupled to the pressure work, which can however not be created from nothing. ISO 14577 
and [1] [2] [3] [4] are incorrect. They required, prescribed, or allowed data fittings and iterations. These missed all of the named unprecedented further applications and cannot even detect the calibration errors in calibration standard indentions of [1] (five of the six standards with calibration error; two mix-ups of figure designations; ignoring phase-transitions). These experimental errors are not corrected and still used in ISO 14577 documents. The unprecedented novelties have already been applied for conical/pyramidal/wedged indentations [5] [6] [13] [22] [23] [24] [25], and further applications are expected for the Equations (2), (7) and (8) when ISO 14577 will be profoundly revised on sound physics but not on historical errors. Unfortunately, there are non-scientific problems for a rapid ISO decision, including severe liability questions [23].

\section{Conflicts of Interest}

The author declares no conflicts of interest regarding the publication of this paper.

\section{References}

[1] Oliver, W.C. and Pharr, G.M. (1992) An Improved Technique for Determining Hardness and Elastic Modulus Using Load and Displacement Sensing Indentation Experiments. Journal of Materials Research, 7, 1564-1583. https://doi.org/10.1557/JMR.1992.1564

[2] Fischer-Cripps, A.C. (2002) Nanoindentation. Mechanical Engineering Series. Springer, New York. https://doi.org/10.1007/978-0-387-22462-6

[3] Chudoba, T., Schwaller, P., Rabe, R., Breguet, J.M. and Michler, J. (2006) Comparison of Nanoindentation Results Obtained with Berkovich and Cube Corner Indenters. Philosophical Magazine, 86, 5265-5283. https://doi.org/10.1080/14786430600746424

[4] Oliver, W.C. and Pharr, G.M. (2004) Measurement of Hardness and Elastic Modulus by Instrumented Indentation: Advances in Understanding and Refinements to Methodology. Journal of Materials Research, 19, 3-20. https://doi.org/10.1557/jmr.2004.19.1.3

[5] Kaupp, G. (2013) Penetration Resistance: A New Approach to the Energetics of Indentations. Scanning, 35, 392-401. https://doi.org/10.1002/sca.21080

[6] Kaupp, G. (2017) The ISO Standard 14577 for Mechanics Violates the First Energy Law and Denies Physical Dimensions. Journal of Material Sciences and Engineering, 6, 321-328.

[7] Hertz, H. (1882) Über die Berührung fester elastischer Körper. Journal für die reine und angewandte Mathematik, 92, 156-171. https://doi.org/10.1515/crll.1882.92.156

[8] Hertz, H. (1896) On the Contact of Rigid Elastic Solids and on Hardness. Macmillan and Co., New York. https://archive.org/details/cu31924012500306

[9] Sneddon, I.N. (1965) The Relation between Load and Penetration in the Axisymmetric Boussinesq Problem for a Punch of Arbitrary Profile. International Journal of Engineering Science, 3, 47-57. https://doi.org/10.1016/0020-7225(65)90019-4

[10] Love, A.E.H. (1939) Boussinesq's Problem for a Rigid Cone. The Quarterly Journal of Mathematics (Oxford), 10, 161-175. https://doi.org/10.1093/qmath/os-10.1.161

[11] Kaupp, G. (2016) The Physical Foundation of $F_{\mathrm{N}}=k h^{3 / 2}$ for Conical/Pyramidal In- 
dentation Loading Curves. Scanning, 38, 177-179. https://doi.org/10.1002/sca.21223

[12] Kaupp, G. and Naimi-Jamal, M.R. (2004) Nanoscratching on Surfaces: The Relationships between Lateral Force, Normal Force and Normal Displacement. International Journal of Materials Research, 95, 297-305. https://doi.org/10.3139/146.017952

[13] Kaupp, G. and Naimi-Jamal, M.R. (2013) Penetration Resistance and Penetrability in Pyramidal (Nano)Indentations. Scanning, 35, 111. https://doi.org/10.1002/sca.21038

[14] Wöllmer, S., Zaefferer, S., Göken, M., Mack, T. and Glatzel, T. (2003) Characterization of Phases of Aluminized Nickel Base Superalloys. Surface and Coatings Technology, 167, 83-96. https://doi.org/10.1016/S0257-8972(02)00843-5

[15] Ebenstein, D.M. and Wahl, K.J. (2006) A Comparison of JKR-Based Methods to Analyze Quasi-Static and Dynamic Indentation Force Curves. Journal of Colloid and Interface Science, 298, 652-662. https://doi.org/10.1016/j.jcis.2005.12.062

[16] Johnson, K.L., Kendall, K. and Roberts, A.D. (1971) Surface Energy and the Contact of Elastic Solids. Proceedings of the Royal Society of London. Series A, 324, 301-313. https://doi.org/10.1098/rspa.1971.0141

[17] Ni, G., Cheng, Y.T., Cheng, C.M. and Grummon, D.S. (2004) An Energy-Based Method for Analyzing Instrumented Spherical Indentation Experiments. Journal of Materials Research, 19, 149-157. https://doi.org/10.1557/jmr.2004.19.1.149

[18] Kaupp, G. (2017) Dilemma between Physics and ISO Elastic Indentation Modulus. Journal of Material Sciences and Engineering, 6, 402-405. https://doi.org/10.4172/2169-0022.1000402

[19] Kaupp, G. and Naimi-Jamal, M.R. (2010) The Exponent 3/2 at Pyramidal Nanoindentations. Scanning, 32, 265-281. https://doi.org/10.1002/sca.20206

[20] Malzbender, J. and de With, G. (2002) Indentation Load-Displacement Curve, Plastic Deformation and Energy. Journal of Materials Research, 17, 502-511. https://doi.org/10.1557/JMR.2002.0070

[21] Riccardi, B. and Montanari, R. (2004) Indentation of Metals by a Flat-Ended Cylindrical Punch. Materials Science and Engineering: A, 381, 281-291.

https://doi.org/10.1016/j.msea.2004.04.041

[22] Kaupp, G. (2018) Six Polymorphs of Sodium Chloride upon Depth-Sensing Scanning Macroindentatiom with Unusual Long-Range Cracks Requiring $30 \mathrm{~N}$ Load. Journal of Material Sciences and Engineering, 7, 473-483. https://doi.org/10.4172/2169-0022.1000473

[23] Kaupp, G. (2014) Activation Energy of the Low-Load NaCl Transition from Nanoindentation Loading Curves. Scanning, 36, 582-589. https://doi.org/10.1002/sca.21158

[24] Kaupp, G. (2019) Phase-Transition Energies, New Characterization of Solid Materials and Anisotropy. Advances in Materials Physics and Chemistry, 9, 57-70. https://doi.org/10.4236/ampc.2019.94006

[25] Kaupp, G. (2019) Physical Nanoindentation: From Penetration Resistance to Phase-Transition Energies. Advances in Materials Physics and Chemistry, 9, 103-122. https://doi.org/10.4236/ampc.2019.96009 